J. Lake Sci.(湖泊科学), 2009, 21(6): 768-774

http://www.jlakes.org. E-mail: jlakes@niglas.ac.cn

(C)2009 by Journal of Lake Sciences

\title{
利用薄膜扩散平衡技术分析沉积物间隙水溶解态反应性磷*
}

\author{
时 丹 $^{1,2}$, 丁士明 ${ }^{2 * *}$, 许 笛 $^{2}$, 白秀玲 ${ }^{2}$, 范成新 ${ }^{2}$ \\ (1: 南京农业大学环境工程系, 南京 210093) \\ (2: 中国科学院南京地理与湖泊研究所, 南京 210008)
}

摘 要: 沉积物间隙水溶解态反应性磷(SRP)是反映沉积物磷地球化学特征的敏感指标, 对其高分辨率获取一直是难点. 基于 薄膜扩散平衡(DET)原理，以琼脂糖为原料制备薄膜，通过平衡、切片、提取、测定等步骤，获得溶解态反应性磷的含量信息. 实验确定薄膜在磷溶液中的平衡时间为 $24 \mathrm{~h}$, 通过 $0.25 \mathrm{~mol} / \mathrm{L}$ 硝酸提取 $16 \mathrm{~h}$ 可将薄膜萃取的磷提取完全. 利用DET技术对不同沉 积物间隙水SRP进行了分析，与实际浓度的差异在 $\pm 5 \%$ 以内; 对沉积物剖面的分析结果与Rhizon、微型Peeper等采样技术基本一致, 垂直分辨率可达到 $3 \mathrm{~mm}$ 左右. 利用DET技术对太湖草型和藻型湖区沉积物间隙水SRP进行了分析, 发现草型湖区间隙水剖面SRP 呈峰形分布, 且横向空间分异明显; 藻型湖区间隙水SRP随沉积深度的增加呈升高趋势, 扩散梯度随水温升高而增强.

关键词：薄膜扩散平衡技术; 间隙水; 溶解磷; 高分辨率

\section{Determination of soluble reactive phosphorus in porewaters of sediments using the technique of diffusive equilibration in thin films}

\author{
SHI Dan ${ }^{1,2}$, DING Shiming ${ }^{2}, \mathrm{XU} \mathrm{Di}^{2}$, BAI Xiuling ${ }^{2} \&$ FAN Chengxin ${ }^{2}$ \\ (1: College of Resource and Environmental Science, Nanjing Agriculture University, Nanjing 210093, P.R.China) \\ (2: Nanjing Institue of Geography and Limnology, Chinese Academy of Sciences, Nanjing 210008, P.R.China)
}

Abstract: Distribution of soluble reactive phosphate (SRP) in pore waters is a sensitive indicator showing geochemical properties of phosphorus in sediments. Concentration of SRP in synthetic solutions was obtained based on the principle of diffusive equilibration in thin films (DET). Analytical procedure generally includes equilibration of the gels in solutions or pore waters, section of the gels after retrieval, back elution of phosphorus in the gels, and analysis of SRP in the eluents. Equilibrium and extraction times were determined at $24 \mathrm{~h}$ and $16 \mathrm{~h}$, respectively, with $0.25 \mathrm{~mol} / \mathrm{L} \mathrm{HNO}_{3}$ used as an extractant. Concentrations of SRP in pore waters of different sediments measured by using the established DET technique agree well with its actual values, and the variations are generally within $\pm 5 \%$. Analytical results for sediment profiles with this technique, which has a vertical resolution at about $3 \mathrm{~mm}$, also show comparison with those using sampling techniques of Rhizon and Mini-Peeper. The established DET technique was used to measure SRP profiles in sediments of macrophyte- and algal-dominated regions in Lake Taihu. Peak-shape distribution and horizontal heterogeneity of SRP were observed in macrophyte-dominated region, while an upward and increasing movement of SRP was observed in algal-dominated region with an increase in water temperature.

Keywords: Diffusive equilibration in thin films; pore water; soluble reactive phosphate; high resolution

在淡水系统中, 磷是构成初级生产力和食物链最重要的生源要素, 同时是湖泊富营养化的限制性因 子. 沉积物间隙水磷是指示沉积物磷地球化学特征的敏感指标. 依据间隙水溶解态反应性磷(Soluble Reactive Phosphorus, SRP)的垂向分布，可计算得到该部分磷在沉积物与上覆水体之间的静态交换通量,

* 国家高技术研究发展计划项目(863 计划)(2007AA06Z411)和国家自然科学基金项目(40601087)联合资助. 2009-02-13 收 稿; 2009-04-29 收修改稿. 时丹, 女, 1983 年生, 硕士研究生, E-mail: shidan83@163.com.

** 通讯作者; E-mail: smding@niglas.ac.cn. 
为评价沉积物磷污染特征提供依据.

对间隙水SRP垂向分布的获取依赖于间隙水采集技术的发展. 获取沉积物间隙水样品，有破坏性与 原位采集两种方法. 前者通过破坏沉积物原有的结构获取间隙水，如离心法，压椎法 ${ }^{[1]}$. 该类方法易使沉 积物性质发生变化, 导致分析结果可能有较大的偏差. 原位采集方法是不破坏沉积物结构被动采集间隙 水的方法, 具有代表性的是基于渗透平衡原理的Peeper技术 ${ }^{[2]}$ 、基于负压原理的抽吸技术 ${ }^{[3]}$ 与Rhizon技术 [4]等. 原位采集方法在研究间隙水物化特性方面得到了较多的应用 ${ }^{[5-7]}$. 但是, 空间分辨率低(一般为厘米 级)、平衡时间过长(如普通的Peeper装置需要20d以上)等不足在很大程度上限制了它们的进一步发展. 20 世纪90年代, Davison等 ${ }^{[8]}$ 发展了一种新的原位采集技术一一薄膜扩散平衡技术(Diffusive Equilibration in Thin Films, DET). 该技术的基本原理与Peeper类似，但膜内的水被固定在薄膜凝胶上，外部溶液中的离 子可自由扩散到凝胶中, 通过测定凝胶中不同位置离子的含量获取间隙水剖面的信息 ${ }^{[9]}$. DET在沉积物中 的平衡时间一般不超过 $24 \mathrm{~h}$, 垂直分辨率取决于所采用的分析手段. 对于间隙水中主要的阳离子和阴离 子, 采用常规方法(原子吸收分光光度法、高效液相色谱或离子色谱)的分辨率可精确到 $1 \mathrm{~mm}^{[10]}$; 如采用质 子激发X苂光能谱(PIXE)直接扫描，对金属离子可以获得亚毫米的分辨率 $[9,11]$.

利用 DET 技术获取间隙水的离子含量信息, 通常需要平衡、切片、提取和测定四个步骤. 薄膜中的 阴离子一般采用去离子水进行提取, 该方法能完全回收 $\mathrm{Cl}^{-} 、 \mathrm{SO}_{4}{ }^{2}$ 和 $\mathrm{NO}_{3}{ }^{-}$等低价阴离子, 但对 $\mathrm{PO}_{4}{ }^{3-}$ 的回 收不完全 ${ }^{[10]}$ ，从而限制了该技术对间隙水 SRP 的分析，相关文献的报道也很少 ${ }^{[12-13]}$. 本文利用琼脂糖制 备薄膜, 选择硝酸作为提取剂, 采用酶标仪分析 SRP, 建立了 DET 获取与批量分析间隙水 SRP 的技术, 利用该技术对太湖不同湖区间隙水 SRP 进行了分析.

\section{1 材料与方法}

\section{1 凝胶薄膜制备}

称取 $0.45 \mathrm{~g}$ 琼脂糖(国药集团, 中国), 转移到 $250 \mathrm{ml}$ 三角烧瓶中, 加人 $30 \mathrm{ml}$ 去离子水, 加热溶解, 配 制成浓度为 $1.5 \%$ 的琼脂糖溶液. 趁热用注射器吸取, 注人由两块玻璃板隔成的 $0.4 \mathrm{~mm}$ 厚夹层中. 注人完 成后, 冷却至室温, 将玻璃板浸人去离子水中, 放置 $24 \mathrm{~h}$, 待薄膜与玻璃板自由剥离后, 将薄膜浸人去离 子水中继续浸泡 $24 \mathrm{~h}$, 每隔 $8 \mathrm{~h}$ 换水一次, 随后放人 $0.01 \mathrm{~mol} / \mathrm{L}$ 的 $\mathrm{NaNO}_{3}$ 溶液中浸泡 $24 \mathrm{~h}$ 后备用. 该凝胶 膜含水率大于 $99 \%$, 泡水前后基本不膨胀.

\section{2 提取剂选择}

配制浓度为 $0.1 、 0.5 、 1.0 、 2.0 、 5.0 \mathrm{mg} / \mathrm{L}$ 的磷标准溶液, 将薄膜切成直径为 $2.5 \mathrm{~cm}$ 的圆片, 浸泡到溶 液中, 平衡 $24 \mathrm{~h}$ 后, 取出薄膜, 称重. 分别用去离子水和 $0.25 \mathrm{~mol} / \mathrm{L}$ 的硝酸作为提取剂, 按照质量体积比 1:10 加人提取剂, 10000 转 $/ \mathrm{min}$ 离心 $10 \mathrm{~min}$, 放置 $16 \mathrm{~h}$ 后, 10000 转 $/ \mathrm{min}$ 再次离心 $10 \mathrm{~min}$, 测定提取液中的 磷, 计算两种提取剂的提取率.

\section{3 间隙水 SRP 实测}

考虑到磷标准溶液和间隙水性质的差别, 为验证沉积物间隙水的分析效果, 取不同的湖泊沉积物, 离心获得间隙水, $0.45 \mu \mathrm{m}$ 滤膜过滤. 将薄膜浸泡到 $20 \mathrm{ml}$ 间隙水中, 平衡 $24 \mathrm{~h}$, 按 1.2 步骤用 $0.25 \mathrm{~mol} / \mathrm{L}$ 硝 酸提取, 分别测定平衡后提取液和间隙水中 SRP 的含量, 计算提取率.

\section{4 间隙水 SRP 剖面分析比较}

将琼脂糖制备的薄膜切成 $18.0 \mathrm{~cm}($ 长) $\times 3.5 \mathrm{~cm}$ (宽)尺寸, 平铺于插板上, 边沿保留相同空隙, 将滤膜覆 盖于薄膜上, 通过胶带将孔径为 $0.45 \mu \mathrm{m}$ 、具有生物㤢性的滤膜固定于插板背面, 保持插板正面滤膜的平 整. 将组装的 DET 装置放人去离子水中, 冲氮气 $24 \mathrm{~h}$. 事先从太湖梅梁湾采集两个柱状沉积物样品, 分别 垂直插人一个 DET 装置, 保留界面以上约 $3 \mathrm{~cm}$, 放置 $24 \mathrm{~h}$ 后, 取出装置, 判定水土界面位置. 揭开滤膜, 将薄膜转移至干净玻璃板, 迅速将薄膜切成长约 $3 \mathrm{~cm}$ 的片段, 并进一步切成约 $3 \mathrm{~mm}$ 的小片段. 依次将各 薄膜片段按顺序转移到 $1.5 \mathrm{ml}$ 离心管, 离心管事先已称重, 加人薄膜片段后, 离心管再次称重, 计算得到 各片段的重量, 按质量体积比 $1: 10$ 加人 $0.25 \mathrm{~mol} / \mathrm{L}$ 硝酸, 按上述提取剂选择步骤提取, 用酶标仪(SNY-1 型)分析提取液中 SRP 的含量. 
在插人 DET 装置的同时, 向一个沉积物柱样插人两个微型 Peeper 装置, 分别平衡 $24 \mathrm{~h}$ 和 48h. Peeper 装置的平衡时间取决于小室的厚度和沉积物的性质，垂直分辨率由小室的宽度和小室之间的间隔确定 ${ }^{[2]}$. 本研究使用的微型 Peeper 装置由 54 个间隔 $0.15 \mathrm{~cm}$ 的小室垂直排列组成, 厚度 $0.3 \mathrm{~cm}$, 每一小室体积约为 $225 \mu \mathrm{l}$, 垂直分辨率为 $0.4 \mathrm{~mm}$. 此外, 在另一个沉积物柱样取出 DET 装置后, 用 Rhizon 采样器(Rhizosphere Research Products)采集沉积物不同深度的间隙水. Rhizon 是应用抽吸原理获取间隙水的采样器, 最初应 用于不饱和土壤环境中, 垂直分辨率一般为 $1 \mathrm{~cm}^{[4]}$. 上述间隙水样品经稀释后用酶标仪分析 SRP 的含量.

\section{5 太湖沉积物间隙水磷分析}

2008 年 3 月、 4 月、 5 月中旬分别在太湖梅梁湾(藻型湖区)和东太湖(草型湖区)每样点采集两个柱样, 采样时水温分别在 $9^{\circ} \mathrm{C} 、 16^{\circ} \mathrm{C}$ 和 $21^{\circ} \mathrm{C}$. 沉积物低温保存运回实验室, 放人水循环控温器内, 控制温度与现 场一致. 事先组装 DET 装置, 放人 $0.01 \mathrm{~mol} / \mathrm{L}$ 的 $\mathrm{NaNO}_{3}$ 溶液中, 冲氮气 $24 \mathrm{~h}$. 将 DET 装置插人沉积物, 平 衡 24h, 取出后分析间隙水的 SRP. 根据中国科学院太湖湖泊生态系统研究站监测(2007 年数据), 上述两 个湖区采样点表层沉积物总磷含量分别为 $1830 \mathrm{mg} / \mathrm{kg}$ 和 $517 \mathrm{mg} / \mathrm{kg}$, 水体总磷平均浓度为 $0.24 \mathrm{mg} / \mathrm{L}$ 和 $0.06 \mathrm{mg} / \mathrm{L}$.

\section{6 分析方法}

提取液和间隙水中的 SRP 采用钼蓝比色法, 通过酶标仪微量比色完成. 利用酶标仪分析需要的溶液 体积约 $200 \mu \mathrm{l}$, 可在 $1 \mathrm{~min}$ 内一次性完成 96 个样品的测定 ${ }^{[14]}$. 与传统的分光光度计相比, 该仪器在批量分 析微量体积样品时优势明显, 且测定范围、检出限、检出下限、灵敏度等分析参数没有明显差异(表 1).

表 1 酶标仪和分光光度计分析参数对比

Tab.1 Analytical parameters of spectrophotometer and microphotometry for determination of SRP

\begin{tabular}{cccccc}
\hline & 样品量 $(\mathrm{ml})$ & 测量范围 $(\mathrm{mg} / \mathrm{L})$ & 检出限 $(\mathrm{mg} / \mathrm{L})$ & 检出下限 $(\mathrm{mg} / \mathrm{L})$ & 灵敏度 \\
\hline 分光光度计 & $2-3$ & $0-0.6$ & 0.002 & 0.008 & 0.55 \\
酶标仪 & 0.2 & $0-0.6$ & 0.003 & 0.010 & 0.34 \\
\hline
\end{tabular}

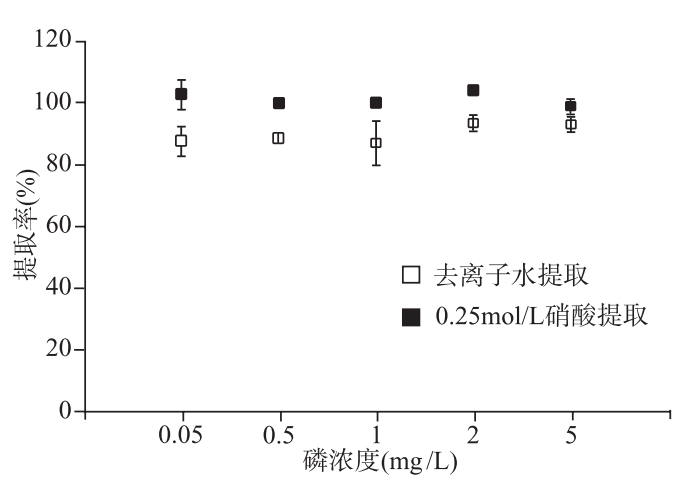

图 1 去离子水和 $0.25 \mathrm{~mol} / \mathrm{L}$ 硝酸提取率对比

Fig.1 Extraction rates of SRP using water and $0.25 \mathrm{~mol} / \mathrm{L} \mathrm{HNO}_{3}$ as extractants

\section{2 结果与讨论}

\section{1 提取剂的选择}

对于 5 种不同浓度的磷溶液, 去离子水的提取 率为 $90 \% \pm 3 \%$, 与 $\mathrm{Krom}^{[10]}$ 等得到的 $89 \% \pm 3 \%$ 一致, 提取率不足说明有少量磷酸根被琼脂糖分子滞留 (图 1). 使用 $0.25 \mathrm{~mol} / \mathrm{L}$ 硝酸的提取率为 $101 \% \pm 2 \%$, 可达到完全回收的效果. 实验确定提取时间为 $16 \mathrm{~h}$ 时, 对于不同浓度的磷溶液均能达到理想的提取效 果. 提取时间过短造成提取率偏低和不稳定, 过长 则影响测定, 提取率偏高. Krom 等 ${ }^{[10]}$ 与 Monbet 等 ${ }^{[12]}$ 用去离子水做提取剂时，提取时间分别为 $2 \mathrm{~h}$ 和 36h, Jarvie 等 ${ }^{[13]}$ 用硫酸做提取剂时，提取时间为 24h. 本研究使用的提取时间, 介于上述研究之间. 进一步提高硝酸浓度，可能会提高提取效率，缩短提取时间，但琼脂膜因酸度过强，在提取过程中产生 碎屑, 给后续的测定带来困难.

\section{2 间隙水 SRP 实测}

收集不同湖泊的 30 个沉积物间隙水样品, SRP 的浓度范围为 $0.01-12.45 \mathrm{mg} / \mathrm{L}$. 利用 $0.25 \mathrm{~mol} / \mathrm{L}$ 硝酸为 提取剂, 通过已确定的平衡时间、提取时间对 30 个沉积物间隙水样品进行了分析, 得到 DET 装置对间隙 水 SRP 的获取率为 $99 \% \pm 6 \%$ (图 2), 表明 DET 可用于间隙水 SRP 的分析. 获取率的不确定性主要来源于 低浓度 SRP 样品. 在提取一步中, 提取剂的加人量是膜体积的 10 倍, 膜萃取的磷被相应稀释 10 倍. 因此, 
通过本研究建立的 DET 技术定量获取间隙水 SRP 的方法下限为 $0.1 \mathrm{mg} / \mathrm{L}$, 当间隙水 SRP 浓度 低于 $0.1 \mathrm{mg} / \mathrm{L}$ 时, DET 的应用受到限制.

\section{3 间隙水剖面分析对比}

利用 DET 装置获得了沉积物间隙水的 SRP 剖面, 同时与 Rhizon 技术、微型 Peeper 的结果进 行了比较. DET 和 Rhizon 获得的 SRP 剖面变化基 本一致(图 3). 由于 Rhizon 的垂直分辨率(约 $1 \mathrm{~cm}$ ) 低于 DET(约 $0.3 \mathrm{~cm})$, 两者的比较不能反映 DET 高分辨率分析效果. 微型 Peeper 的垂直分辨率与 DET 相当(3-4mm). 利用该装置获得的间隙水 $\mathrm{SRP}$ 在平衡 $24 \mathrm{~h}$ 和 $48 \mathrm{~h}$ 后其剖面变化趋势基本一 致, 但是 $48 \mathrm{~h}$ 的变化更加平滑, 据此判断间隙水 SRP 向 Peeper 装置的扩散在 48h 内达到平衡. 该

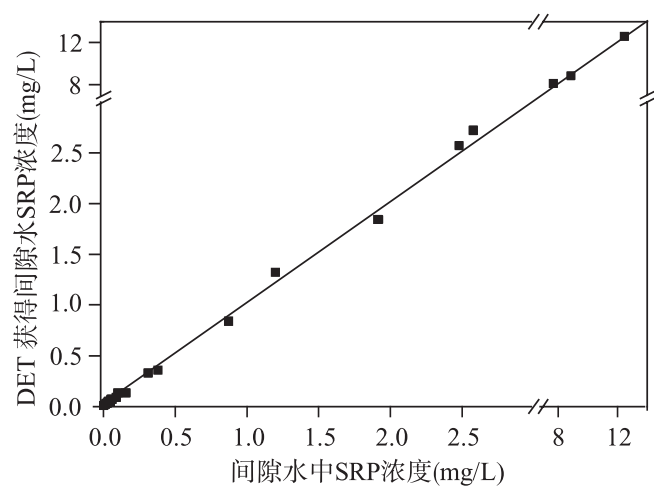

图 2 沉积物间隙水和提取液中 SRP 含量的相关关系

Fig.2 Relationship between SRP concentration in porewaters and that extracted by DET Peeper 装置平衡时间显著短于文献报道的同类装置 ${ }^{[2]}$. 与微型 Peeper 相比, 通过 DET 获得的 SRP 含量变 化趋势一致, 在水土界面至沉积深度 $8 \mathrm{~cm}$ 处出现向下增加的趋势, 随后保持稳定. 然而, DET 获得的 SRP 梯度变化有一定程度的变缓. 该变化可能是磷在薄膜内的侧向扩散造成. 与 Peeper 不同, DET 膜是由自 由贯通的凝胶组成, 相互之间没有间隔. DET装置从沉积物中取出后, 在 SRP 有浓度梯度的情况下, 离子 会从高浓度向低浓度发生侧向扩散, 所获得的剖面信息出现一定程度的失真 ${ }^{[15]}$. 缩短装置取出后至切成 小片段的时间，可以降低侧向扩散. 目前已研制出一种限制型 DET 装置, 与 Peeper 装置类似, 凝胶膜被 隔成 $0.2 \mu \mathrm{m}$ 凝胶片段, 但是样品体积少, 应用时需要精度更高的分析技术 ${ }^{[16]}$. 上述现象也可能是操作方 式不当造成的. DET 装置从沉积物中取出后, 采用 Krom 等 ${ }^{[10]}$ 的方法, 用去离子水冲洗以去除表面附着的 沉积物. Mortimer 等 ${ }^{[17]}$ 发现该方法在测定海洋沉积物间隙水时可能因间隙水稀释而产生误差, 用湿润薄 纸擦拭更合适.
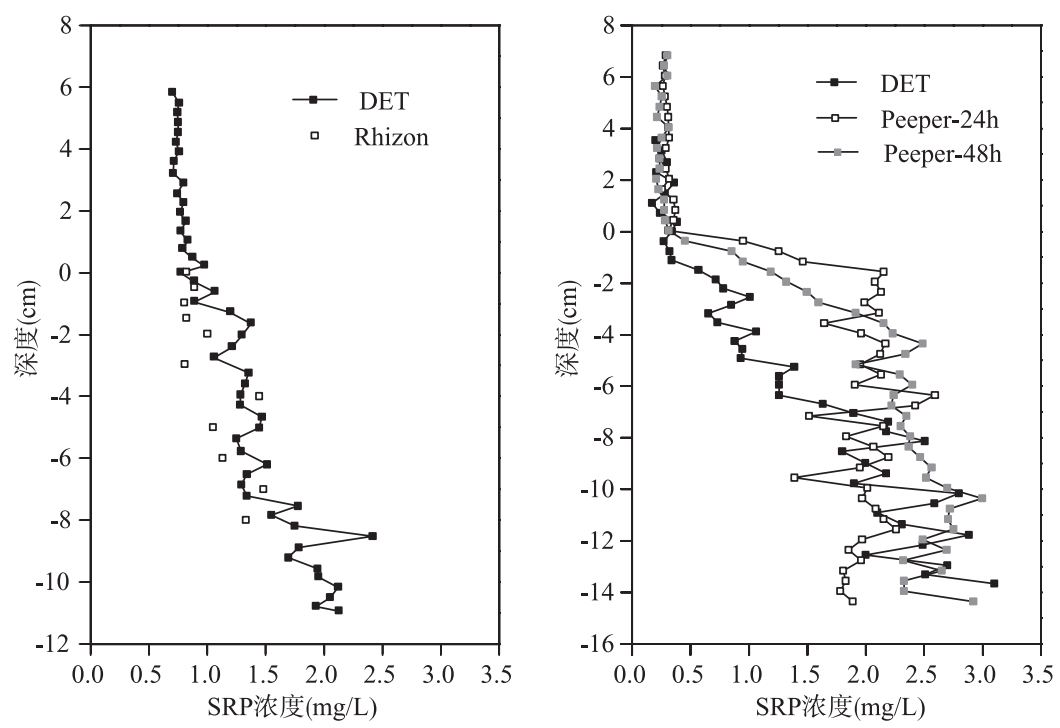

图 3 DET、Rhizon 与微型 Peeper 技术获取沉积物间隙水 SRP 的垂向分布对比

Fig.3 Comparison of SRP concentration profiles in porewaters detected by DET with those by Rhizon and Mini-peeper 


\section{4 太湖沉积物间隙水 SRP 的垂向分布特征}

利用DET技术获得东太湖草型区间隙水SRP含量的剖面分布(图4). 从3-5月, 伴随水温的升高, 剖面 $\mathrm{SRP}$ 平均含量分别为 $0.27 、 0.36 、 1.21 \mathrm{mg} / \mathrm{L}$ ，也呈现升高的趋势. 在 3 月的 0 至 $-4 \mathrm{~cm}$ 深度、 4 月的 0 至 $-9 \mathrm{~cm}$ 深 度, 以及 5 月的 0 至 $-8 \mathrm{~cm}$ 深度, 剖面出现峰形分布. 其中, 3 月 SRP浓度峰值出现在 $-2 \mathrm{~cm}$ 附近, 4月和5月峰 值均在-4cm附近. 但是，在SRP峰形分布区同一时期两个平行剖面之间有着明显的差异. 例如，三个时 期两个平行剖面中 $S R P$ 峰值高的分别为 $0.70 \mathrm{mg} / \mathrm{L} 、 1.35 \mathrm{mg} / \mathrm{L} 、 3.45 \mathrm{mg} / \mathrm{L}$, 分别是低值的 $1.8 、 2.1$ 和1.5倍. 间 隙水离子在横向空间上分布不均的特点也被其他研究者观察到 ${ }^{[7]}$. Shuttleworth等 ${ }^{[18]}$ 与 Devries 等 ${ }^{[19]}$ 的研究 发现, 间隙水中的离子在剖面中的分布具有可观的 “空间异质性”; 当分辨率精确到毫米量级时, 离子在 垂向分布上可能有小尺度的“突起”或“峰形”，而横向不同位置则出现比较大的差异，推测与易降解有机 质在沉积物表层分布不均造成不同的矿化强度有关 ${ }^{[19-20]}$. 浅水湖泊较为强烈的风浪扰动可使得不同性质 的颗粒物充分混匀, 降低空间异质性; 生物扰动则造成颗粒物重新分布, 加大空间异质性. 本研究获得 的间隙水剖面处于草型湖区, 且剖面峰形的分布区与水生植物根系分布较为一致, 因此上述剖面分布特 征可能由根系活动造成, 主要由两种过程主导. 一方面, 水生植物根系直接从沉积物中吸收磷, 降低间 隙水中磷的浓度; 同时, 通过根系释放的氧气提高沉积物的氧化还原电位, 增强沉积物固相对磷的吸附, 也起到降低或稳定间隙水磷的作用 ${ }^{[21]}$. 另一方面, 水生植物死亡分解, 可直接或间接释放磷, 增加间隙 水磷的含量 ${ }^{[22]}$. 运用Fick第一定律计算界面扩散通量，3、4、5月分别为 $0.18 \mathrm{mg} /\left(\mathrm{m}^{2} \cdot \mathrm{d}\right) 、 0.85 \mathrm{mg} /\left(\mathrm{m}^{2} \cdot \mathrm{d}\right)$ 、 $1.03 \mathrm{mg} /\left(\mathrm{m}^{2} \cdot \mathrm{d}\right)$, 说明该湖区SRP的界面交换通量以释放为主, 并且随温度的升高有增加的趋势. 据此推测 在东太湖藻型湖区, 后一作用可能占主导地位. 由于该湖区水柱中磷的浓度显著低于其他湖区, 说明水 生植物从水柱中吸收磷对维持该湖区较低的营养水平起到重要的作用.
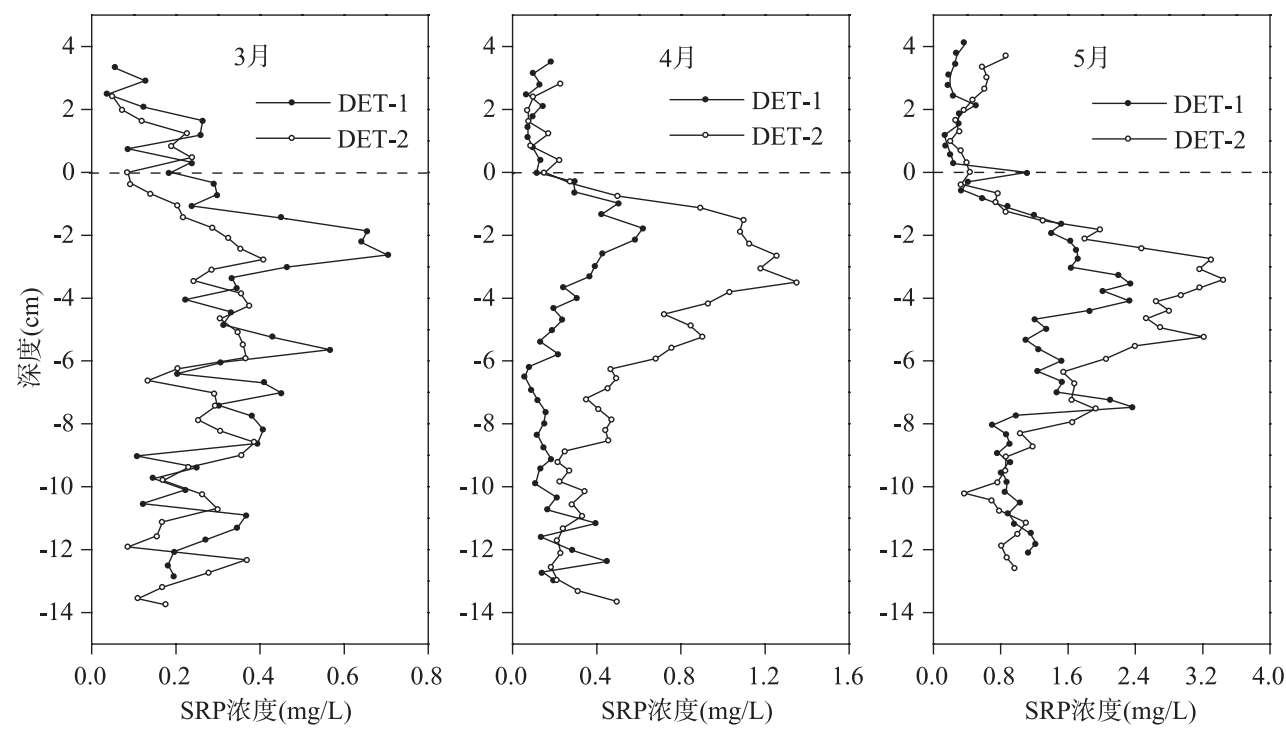

图 4 东太湖沉积物间隙水 SRP 的垂向分布(DET-1 和 DET-2 为同一柱样的两个平行)

Fig.4 Concentration of SRP in porewaters sampled from East Lake Taihu

利用DET装置对太湖藻型区间隙水SRP进行了分析(图5). 从3月到5月，两个平行的DET剖面分布较 为一致, 说明DET操作方法可行, 从而排除了去离子水冲洗DET装置表面是造成SRP梯度变缓的原因. 随 水温的升高, SRP含量的平均值从 3 月的 $0.57 \mathrm{mg} / \mathrm{L} 、 4$ 月的 $0.93 \mathrm{mg} / \mathrm{L}$ 升高到 5 月的 $2.17 \mathrm{mg} / \mathrm{L}$. 同时， 3 个时期 $\mathrm{SRP}$ 含量均出现随深度逐渐升高的趋势, 3月和 4 月份升高的拐点出现在 $-6 \mathrm{~cm}$ 处, 而 5 月份在 $-2 \mathrm{~cm}$ 处, 梯度 范围明显扩大. 运用Fick第一定律计算界面扩散通量，3、4、5月分别为 $0.058 \mathrm{mg} /\left(\mathrm{m}^{2} \cdot \mathrm{d}\right) 、 0.422 \mathrm{mg} /\left(\mathrm{m}^{2} \cdot \mathrm{d}\right)$ 
和 $0.553 \mathrm{mg} /\left(\mathrm{m}^{2} \cdot \mathrm{d}\right)$, 说明 $\mathrm{SRP}$ 的界面交换通量以释放为主，且随水温升高释放潜力有增大的趋势. 已有研 究表明, 沉积物向水体释放磷以铁磷和活性有机磷为主 ${ }^{[23]}$, 并存在季节性释放机制 ${ }^{[24]}$. 在春季开始, 水 温升高提高好氧微生物活性, 促进有机质的降解和有机磷的矿化; 随着沉积物表层氧含量的降低, 沉积 物向戻氧环境转变, 造成铁氢氧化物和氧化物的还原, 导致铁磷的释放和向上迁移. 上述过程在藻型湖 区尤为强烈 ${ }^{[25-26]}$. 蓝藻作为自生性有机质, 具有比外源输人有机质更强的可降解性. 当大规模蓝藻爆发 后, 大量残体沉积于沉积物表层, 并经历冬季低温储存. 在春季水温升高后, 蓝藻残体降解逐渐加剧, 造 成局部还原环境. 在此条件下, 硫还原细菌活性增强, 促使大量 $\mathrm{S}^{2-}$ 产生, 与 $\mathrm{Fe}^{2+}$ 生成FeS或 $\mathrm{FeS}_{2}$ 沉淀, 降 低由于 $\mathrm{Fe}^{2+}$ 的重新氧化在界面附近形成的二次吸附, 促使铁磷的大量溶解和向水体释放. 上述过程一直 持续到夏季, 可能是造成夏季藻型区上覆水含量显著升高的主要原因之一 ${ }^{[26]}$, 同时为蓝藻的生长和持续 爆发提供必要的磷源 $[24]$.
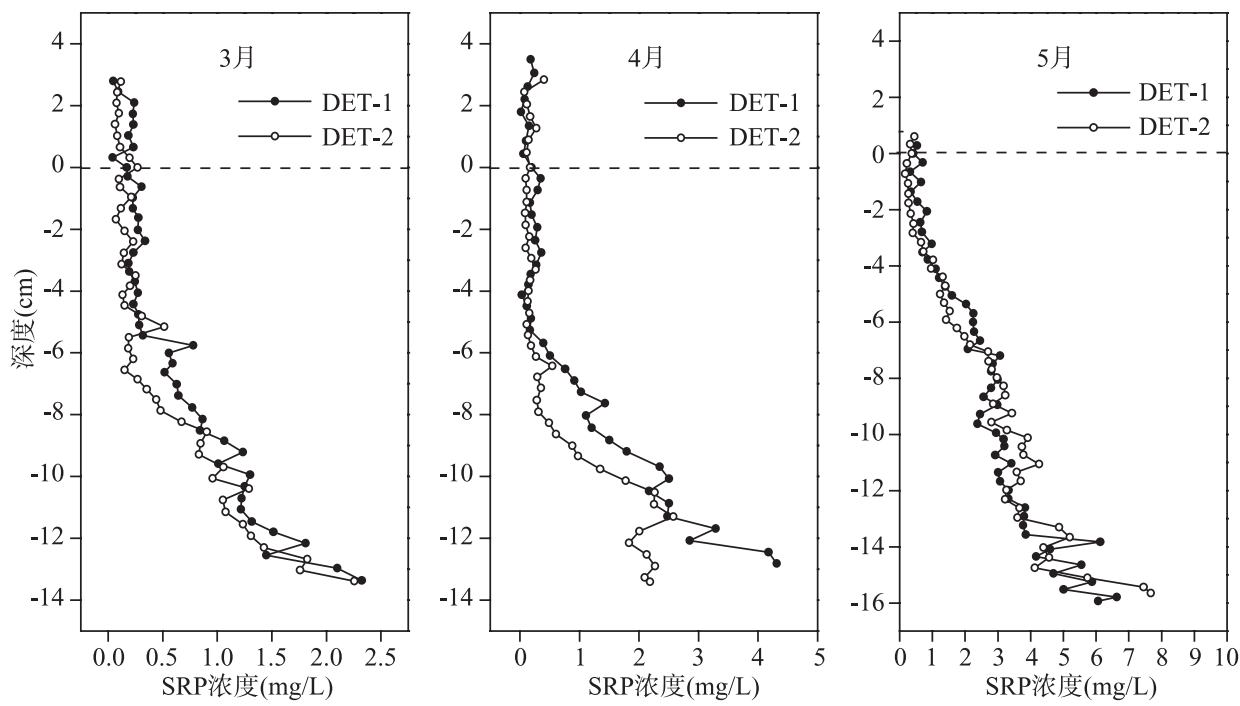

图 5 梅梁湾藻型区沉积物间隙水 SRP 的垂向分布(DET-1 和 DET-2 为同一柱样的两个平行)

Fig.5 Concentration of SRP in porewaters sampled from algal-dominated region in Meiliang Bay of Lake Taihu

\section{3 结论}

根据 DET 技术原理，使用常规的琼脂糖制作凝胶薄膜，通过薄膜平衡、切片、酸提取、酶标仪测定 等步骤，获得沉积物间隙水中的 SRP 含量信息. 实验确定薄膜在磷溶液中的平衡时间为 $24 \mathrm{~h}$ ，通过 $0.25 \mathrm{~mol} / \mathrm{L}$ 硝酸提取 $16 \mathrm{~h}$ 可将薄膜萃取的 SRP 提取完全. 对沉积物剖面的分析结果与 Rhizon、微型 Peeper 等采样技术基本一致. 利用DET获取太湖草型和藻型区沉积物间隙水 SRP 的垂向分布信息, 结果能够较 好的反映间隙水 SRP 的时空分布特征.

总体而言，本研究所建立的DET测定间隙水SRP方法所需的平衡和提取时间较短，通过酶标仪微量 比色可在一般实验室实现大量样品的快速分析，获得的SRP垂直分辨率可提高到mm级，与现有方法相比 优势十分明显.

致谢: 感谢匿名审稿专家提出的宝贵意见.

\section{4 参考文献}

[1] 刘素美, 张 经. 沉积物间隙水的几种制备方法. 海洋环境科学, 1999, 18(2): 66-71.

[2] Hesslein RG. An in situ sampler for close interval pore water studies. Limnology and Oceanography, 1976, 21: 912-914. 
[3] Bertolin A, Rudello D, Ugo P. A new device for in-situ pore-water sampling. Marine Chemistry, 1995, 49: $233-239$.

[4] Seeberg-Elverfeldt J, Schluter M, Feseker T et al. Rhizon sampling of porewaters near the sediment-water interface of aquatic systems. Limnology and Oceanography-Methods, 2005, 3: 361-371.

[5] Fan CX, Zhang L, Wang JJ et al. Processes and mechanism of effects of sludge dredging on internal source release in lakes Chinese Science Bulletin, 2004, 49(17): 1853-1859.

[6] 王建军, 范成新, 张 路等. 太湖底泥间隙水中金属离子分布特征及相关性. 中国环境科学, 2004, 24(1): 120-124.

[7] 张 路, 范成新, 王建军. 太湖草藻型湖区间隙水理化特性比较. 中国环境科学, 2004, 24(5): 556-560.

[8] Davison W, Grime GW, Morgan JAW et al. Distribution of dissolved iron in sediment pore waters at submillimetre resolution. Nature, 1991, 352: 323-325.

[9] Davison W, Zhang H, Grime GW. Characteristics of gel probe used for measuring the chemistry of pore waters. Environmental Science \& Technology, 1994, 28: 1623-1632.

[10] Krom MD, Davison P, Zhang H et al. High resolution pore-water sampling with a gel sampler. Limnology and Oceanography, 1994, 39: 1967-1972.

[11] Fones GR, Davison W, Grime GW. Development of constrained DET for measurements of dissolved iron in surface sediments at sub-mm resolution. The Science of the Total Environment, 1998, 221: 127-137.

[12] Monbet P, McKelvie ID, Worsfold PJ. Combined gel probes for the in situ determination of dissolved reactive phosphorus in porewaters and characterization of sediment reactivity. Environmental Science \& Technology, 2008, 42: 5112-5117.

[13] Jarvie HP, Mortimer RJG, Palmer-Felgate EJ et al. Measurement of soluble reactive phosphorus concentration profiles and fluxes in river-bed sediments using DET get probes. Journal of Hydrology, 2008, 350: 261-273.

[14] Laskov C, Herzog C, Lewandowski J et al. Miniaturized photometrical methods for the rapid analysis of phosphate, ammonium, ferrous iron, and sulfate in pore water of freshwater sediments. Limnology and Oceanography-Methods, 2007, 5: 63-71.

[15] Harper MP, Davison W, Tych W. Temporal, spatial, and resolution constraints for in situ sampling devices using diffusional equilibration: Dialysis and DET. Environmental Science \& Technology, 1997, 31: 3110-3119.

[16] Docekalov H, Clarisse O, Salomon S et al. Use of constrained DET probe for a high-resolution determination of metals and anions distribution in the sediment pore water. Talanta, 2002, 57: 145-155.

[17] Mortimer RJG., Krom MD, Hall POJ et al. Use of gel probes for the determination of high resolution solute distributions in marine and estuarine pore waters. Marine Chemistry, 1998, 63: 119-129.

[18] Shultt1ewo SM, Davison W, Taylor JH. Two-dimensional and fine structure in the concentrations of iron and manganese in sediment pore-waters. Environmental Science \& Technology, 1999, 33: 4169-4175.

[19] Devries CR, Wang FY. In situ two-dimensional high-resolution profiling of sulfide in sediment interstitial waters. Environmental Science \& Technology, 2003, 37: 792-797.

[20] Lehtoranta J, Ekholm P, Pitkänen H. Eutrophication-driven sediment microbial processes can explain the regional variation in phosphorus concentrations between Baltic Sea sub-basins. Journal of Marine Systems, 2008, 74: 495-504.

[21] Aldridge KT, Ganf GG. Modification of sediment redox potential by three contrasting macrophytes: implications for phosphorus adsorption/desorption. Marine and Freshwater Research, 2003, 54: 87-94.

[22] Farmer JG, Bailey-Watts AE, Kirika A et al. Phosphorus fractionation and mobility in loch leven sediments. Aquatic Conservation-Marine and Freshwater Ecosystems, 1994, 4: 45-56.

[23] Rydin E. Potentially mobile phosphorus in Lake Erken sediment. Water Research, 2000, 34: 2037-2042.

[24] Rozan TF, Taillefert M, Trouwborst RE et al. Iron-sulfur-phosphorus cycling in the sediments of a shallow coastal bay: Implications for sediment nutrient release and benthic macroalgal blooms. Limnology and Oceanography, 2002, 47: 1346-1354.

[25] Lewandowski J, Ruter K, Hupfer M. Two-dimensional small-scale variability of pore water phosphate in freshwater lakes: Results from a novel dialysis sampler. Environmental Science \& Technology, 2002, 36: 2039-2047.

[26] Spears BM, Carvalho L, Perkins R et al. Sediment phosphorus cycling in a large shallow lake: spatio-temporal variation in phosphorus pools and release. Hydrobiologia, 2007, 584: 37-48. 